\title{
Characterization of Fluctuations and Electron Transport in Two-Dimensional Simulations of Hall Thrusters using an Axial-Azimuthal Hybrid Model
}

\author{
By Eduardo Fernandez, Caleb Dowdy and Jacob Aley \\ Department of Mathematics and Physics, Eckerd College, Saint Petersburg, Florida, USA
}

(Received August 7th, 2015)

\begin{abstract}
A two-dimensional model of the Hall thruster with kinetic, non-magnetized ions, and fluid electrons is presented. The model dynamically evolves azimuthal flows and fluctuations, differing from standard hybrid models that assume axisymmetry and resolve quantities in the radial and axial coordinates only. Unlike those descriptions, which typically use adhoc cross-field electron transport parameters in order to sustain the discharge, the present model relies on classical transport and fluctuations generated within the plasma. A number of low-frequency wave modes are captured in the simulation, from the lowest (few kHz) "breathing mode", to waves on the order of a few hundred kHz. At issue is the characterization of the various modes with regards to their instability mechanisms, their spectral signatures, their dependence on plasma inhomogeneity along the channel, and their role in cross-field electron transport. Simulations show that gradient-driven drift instabilities emerge downstream of the peak of the magnetic field, as predicted by linear stability analysis, while strong, ionization driven fluctuations take place upstream. While fluctuations result in fluctuation-driven transport, they do not drive sufficient current to match experimental measurements. Electron transport is reduced in the strong magnetic field region to near classical levels, in qualitative agreement with experiments.
\end{abstract}

Key Words: Hall Thruster, Anomalous Transport, Hybrid Simulations

\section{Nomenclature}

$\theta \quad:$ azimuthal coordinate direction

$z \quad:$ axial coordinate direction

$u_{e z} \quad: \quad$ axial electron velocity

$u_{e \theta} \quad: \quad$ azimuthal electron velocity

$\phi \quad:$ electrostatic potential

$E \quad$ : electric field

$\mu_{\perp} \quad$ : classical perpendicular electron mobility

$\nu_{e n}:$ electron neutral collision frequency

$\omega_{c e} \quad$ : electron cyclotron frequency

$B_{r} \quad$ : radial component of magnetic field

$D_{\perp} \quad$ : classical perpendicular diffusion coefficient

$e \quad$ : magnitude of electron charge

$k \quad$ : Boltzmann constant

$n_{e} \quad:$ electron number density

$T_{e} \quad: \quad$ electron temperature

$u_{i} \quad$ : ion velocity

$u_{n} \quad:$ neutral velocity

$L \quad$ : length of ionization zone

\section{Introduction}

While the basic operation of Hall thrusters is well understood, key physics issues remain open for study. Among those, crossfield electron transport (transport perpendicular to magnetic field lines) stands as one of the most important. The crossfield transport that is predicted from electron-neutral collisions is not sufficient to explain the observed current. ${ }^{1,2)}$ Along with wall effects, fluctuations have been proposed as a candidate for the enhanced plasma conductivity. Indeed, theoretical analysis predicts a number of unstable wave modes in the Hall thruster plasma. ${ }^{3)}$ In addition, fluctuations over a wide frequency and wavenumber range have been experimentally measured. ${ }^{4)}$ In this paper we study the Hall thruster plasma via numerical simulation using a hybrid fluid, Particle-In-Cell (PIC) method aimed at resolving low frequency, long wavelength phenomena. Typical hybrid Hall thruster models are in the axial ( $\mathrm{z}$ ) and radial (r) coordinates, hereby referred to as r-z models, assuming axi-symmetry in order to circumvent the azimuthal direction. The novel feature of our hybrid model is that it is in the axial and azimuthal $(\theta)$ directions, and as such it treats the azimuthal physics self-consistently. On the other hand, important physics pertaining to the radial direction, including radial losses and wall effects are absent in our model. The objective is to construct as simple a model as possible with special focus on the azimuthal dynamics.

Hybrid simulation models have been used by a variety of groups to study Hall thrusters. ${ }^{5-7)}$ Such simulations have been successful at capturing the basic plasma equilibrium as well as basic wave modes, including the so-called "breathing mode". Hybrid models treat the heavy species (xenon neutrals and ions) as particles while the electrons are treated as a fluid. A key feature of $\mathrm{r}-\mathrm{z}$ models is the need to use an ad-hoc electron mobility in the cross-field electron momentum equation. Simply using the so-called "classical" mobility based on electron-neutral collisions does not sustain the discharge - the 
plasma does not "ignite" so-to-speak. This is consistent with the experimental finding that the discharge current is larger than can be accounted for by collisions alone. ${ }^{2)}$

In this work we use a hybrid ( $\mathrm{z}-\theta$ ) model without the use of ad-hoc transport parameters. A basic description of the model is given in section 2, along with the solution method employed. In section 3 the simulation results are presented. First, the basic plasma equilibrium is discussed. Secondly, the fluctuations that emerge in the model are examined. Such fluctuations are studied first in the linear regime as well as in the fully saturated, steady state regime. The character of the observed disturbances is analyzed in terms of their spectral signatures, their dependence on axial position, and their contribution to electron transport. Section 4 presents the conclusions of the paper.

\section{Model}

The basic description of the $\mathrm{z}-\theta$ model has been presented elsewhere ${ }^{8)}$ here we will outline its main features. The guiding principles are a) to construct a model close in structure to the workhorse $\mathrm{r}-\mathrm{z}$ hybrid models in use today by various research groups, b) to make the model as simple as possible in order to focus on the azimuthal dynamics, and c) to capture the low-frequency plasma behavior of the entire thruster (from the anode to the near field, including the full azimuth). To this end, the model resolves the plasma in the two-dimensional axial- azimuthal plane, with the $\theta$ direction being the periodic direction, as shown in Fig. 1 (if preferred, one can view the computational domain as a Cartesian x-y plane with one of the directions being periodic). The chosen thruster geometry is that of the Stanford Hall Thruster (SHT), a laboratory thruster with a channel length of $8 \mathrm{~cm} .{ }^{2,9)}$ In the simulation the anode forms one of the axial boundaries at $\mathrm{z}=0 \mathrm{~cm}$, with the other axial computational boundary at $\mathrm{z}=12 \mathrm{~cm}, 4 \mathrm{~cm}$ past the channel exit plane. In the periodic direction, the full azimuth is covered, consistent with the aim of this work to resolve long wavelengths $(\geq 1 \mathrm{~cm})$. (This contrasts with some kinetic simulations where only an angular sector is resolved. $)^{10}$ In the axial direction, the peaked magnetic field around the channel exit imposes an inhomogeneity in the device. As a result, we employ a non-uniform grid in the axial coordinate of 40 grid points (the grid clusters its points around the peak in the magnetic field, the region where the gradients are expected to be largest). In the $\theta$ direction, we use 50 grid points, which gives an azimuthal grid spacing of $0.5 \mathrm{~cm}$ (consistent with a mean channel radius of $4.1 \mathrm{~cm}$ for the SHT). The magnetic field is assumed to be a static field, purely in the radial direction, and thus perpendicular to the computational plane. It is obtained from experimental SHT measurements. ${ }^{9)}$ As in standard r-z models, the z- $\theta$ model treats the ions and neutrals via a PIC method. However, unlike r-z descriptions, the $\mathrm{z}-\theta$ model studied here does not treat wall particle collisions. Xenon neutral and ion superparticles are initialized randomly in the domain with their axial and azimuthal velocities obtained by inverting a Maxwellian distribution. Such particles stay in the computational domain unless they exit the computational axial boundaries. Xenon neutrals are injected at the anode given the experimentally obtained mass flow rate of $2 \mathrm{mg} / \mathrm{s}$, with velocities obtained by inverting a Maxwellian distribution flux. Since the injected neutrals do not encounter walls, they can only move forward axially. We point out that this is a significant difference with respect to r-z hybrid models in which neutrals collide with the channel walls and are then diffusely injected back in the domain, leading to a more peaked neutral density profile. (The omission of wall-effects cannot be overstated, but it is in keeping with our goals of retaining simplicity and focus on azimuthal fluctuations.) Similarly, in order to simplify the physics, other collisions like charge-exchange and elastic momentum transfer are likewise omitted. The only collisions kept in the model are ionizing collisions: at each timestep, ion superparticles are born in each computational cell according to the instantaneous, local ionization rate using a standard MonteCarlo method. The form of the temperature-dependent ionization rate used here is that suggested by Ahedo, ${ }^{11)}$ obtained from fits to measured ionization collision cross-sections averaged over a Maxwellian distribution. Ion superparticles only respond to the instantaneous electric field. A standard leapfrog method is used to advance the ion and neutral superparticles. The ion and neutral particle positions yield, via bi-linear interpolation to the grid points, ion and neutral densities respectively. Such densities are then used by the fluid equations in order to find the electrostatic potential and the other quantities associated with the electron fluid. Quasineutrality is imposed, in keeping with our goal of resolving long wavelengths. As a result, the ion density obtained from the PIC method automatically yields the electron density.

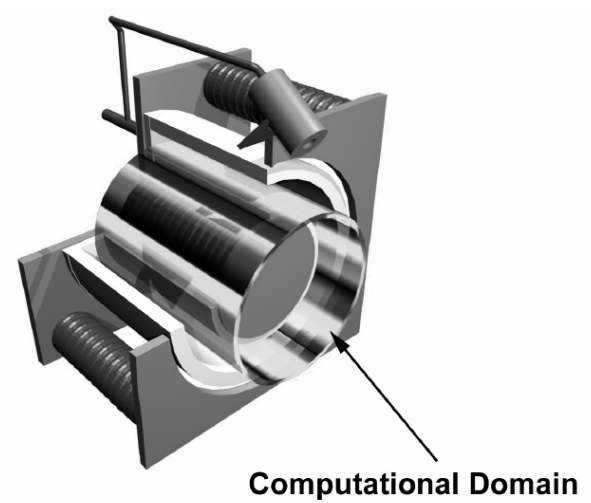

Fig. 1. Schematic of the thruster modeled and the computational domain.

The electron fluid is described by continuity, momentum and energy conservation equations. The electron momentum has an axial as well as an azimuthal component. As it is done in $\mathrm{r}-\mathrm{z}$ models, electron inertia is omitted as we do not intend to resolve frequencies on the order of the Larmor frequency. As a result, the axial and azimuthal electron momentum equations become: ${ }^{8)}$ 
$u_{e_{z}}=-\mu_{\perp} E_{z}-\frac{D_{\perp}}{n_{e}} \frac{\partial n_{e}}{\partial z}-\frac{D_{\perp}}{T_{e}} \frac{\partial T_{e}}{\partial z}-\frac{1}{1+\left(\frac{v_{e n}}{\omega_{c e}}\right)^{2}} \frac{E_{\theta}}{B_{r}}-\frac{1}{1+\left(\frac{v_{e n}}{\omega_{c e}}\right)^{2}} \frac{k T_{e}}{e n_{e} B_{r} r} \frac{\partial n_{e}}{\partial \theta}$

$u_{e_{\theta}}=-\mu_{\perp} E_{\theta}-\frac{D_{\perp}}{n_{e} r} \frac{\partial n_{e}}{\partial \theta}+\frac{1}{1+\left(\frac{v_{e n}}{\omega_{c e}}\right)^{2}} \frac{E_{z}}{B_{r}}+\frac{1}{1+\left(\frac{v_{e n}}{\omega_{c e}}\right)^{2}} \frac{k T_{e}}{e n_{e} B_{r}} \frac{\partial n_{e}}{\partial z}+\frac{1}{1+\left(\frac{v_{e n}}{\omega_{c e}}\right)^{2}} \frac{k}{e B_{r}} \frac{\partial T_{e}}{\partial z}$

Note the EXB and diamagnetic drift components (fourth and fifth terms in Eq. (1) respectively) would be missing in $r-z$ models. If these terms in $u_{e z}$ are large enough and properly correlated with the plasma density they will result in significant electron transport (we will see in section 3 below that this is indeed the case). The electron energy equation is given by

$\frac{3}{2} n_{e} k\left(\frac{\partial T_{e}}{\partial t}+\vec{u}_{e} \cdot \nabla T_{e}\right)+n_{e} k T_{e} \nabla \cdot \vec{u}_{e}-\nabla \cdot \vec{q}_{e}=S_{\text {joule }}-S_{\text {ioniz }}$

with $S_{\text {joule }}=m_{e} n_{e} v_{\text {en }}\left\|\overrightarrow{u_{e}}\right\|^{2}$ and $S_{\text {ioniz }}=\dot{n}_{e} e_{i} k+\dot{n}_{e} \frac{3}{2} k T$

giving the joule heating and ionization loss terms respectively. The thermal heat flux on the left-hand side is given by the classical thermal diffusivity and does not include Bohmenhanced diffusion as it is the case in some r-z models. ${ }^{5}$ Finally, no wall losses are used in our description consistent with our early assumption of neglecting wall effects (admittedly, as was mentioned above, this is a considerable simplification). To simplify further, in the model the electron temperature is averaged over the azimuthal direction in order to yield a onedimensional (axial) energy equation; this is in some sense analogous to what is done in r-z models, where the temperature equation is averaged along the magnetic field contours in order to yield a quasi-1D temperature equation. Such time-dependent electron equation is solved via a Runge-Kutta scheme, with a timestep at least 100 times smaller than the heavy particle timestep. The electric field is assumed electrostatic (consistent with a low-beta plasma), and thus given by $\vec{E}=-\nabla \phi$, where $\phi$ is the electrostatic electric potential. Such electrostatic potential is not solved via Poisson's equation. Instead, it is obtained by combining current continuity, electron momentum, and quasineutrality. The result is a convectiondiffusion equation ${ }^{8)}$ which is discretized via a finite difference scheme, resulting in a block-tridiagonal system of equations which are solved via a direct-solve method. After an initial condition of a given electron temperature and a given distribution of particles in the domain, the electric field in calculated. The simulation enters into its time-advance loop where particles are moved using the calculated electric field, xenon superparticles are ionized (according to the ionization rate), xenon superparticles are injected into the domain (according to the mass flow rate), and new particle densities are then calculated using the PIC method. Next, the electron temperature and electric field are calculated, and then simulation loop starts again until a steady state is achieved and the longest (breathing-mode) time scales are resolved.

As explained in an earlier publication, ${ }^{8}$ special care must be taken when solving for the electric potential. Such equation uses particle densities (ion and neutral), as well as their spatial derivatives. Since particle data is inherently noisy, the bilinearly interpolated particle density results in numerical instability when solving for the electric potential. A solution to this problem was found by applying a smoothing spline filter to the PIC obtained densities in order to remove the noise and render smooth coefficients in the electric potential equation. In fully kinetic approaches the electric potential is found from Poisson's equation, wherein the particle density does not enter as a derivative and the PIC approach suffices. In our hybrid model spatial derivatives of PIC derived quantities enter in the potential calculation, and thus the need for extra smoothing. Further details of the numerical method and its merits/limitations can be found in earlier work. ${ }^{8)}$

\section{Results}

\subsection{Plasma equilibrium}

Given the relative simplicity of the formulation the question arises as to whether the model can yield a plasma equilibrium that is at least qualitatively similar to that measured in the SHT. Fig. 2 shows the time evolution, spanning $1.5 \mathrm{~ms}$, of the discharge current for a 200 volt simulation with a mass flow rate of $2 \mathrm{mg} / \mathrm{s}$. After an early transient a sustained, quasi steady state ensues, with periodic "breathing mode" cycles of about 3 $\mathrm{kHz}$.

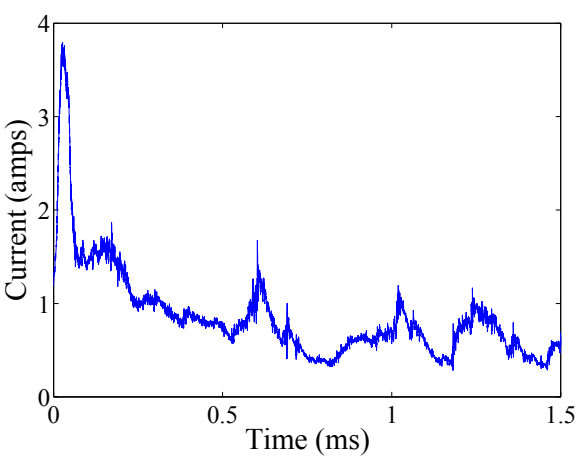

Fig. 2. Discharge current evolution for a $200 \mathrm{~V}$ simulation, covering $1.5 \mathrm{~ms}$. 

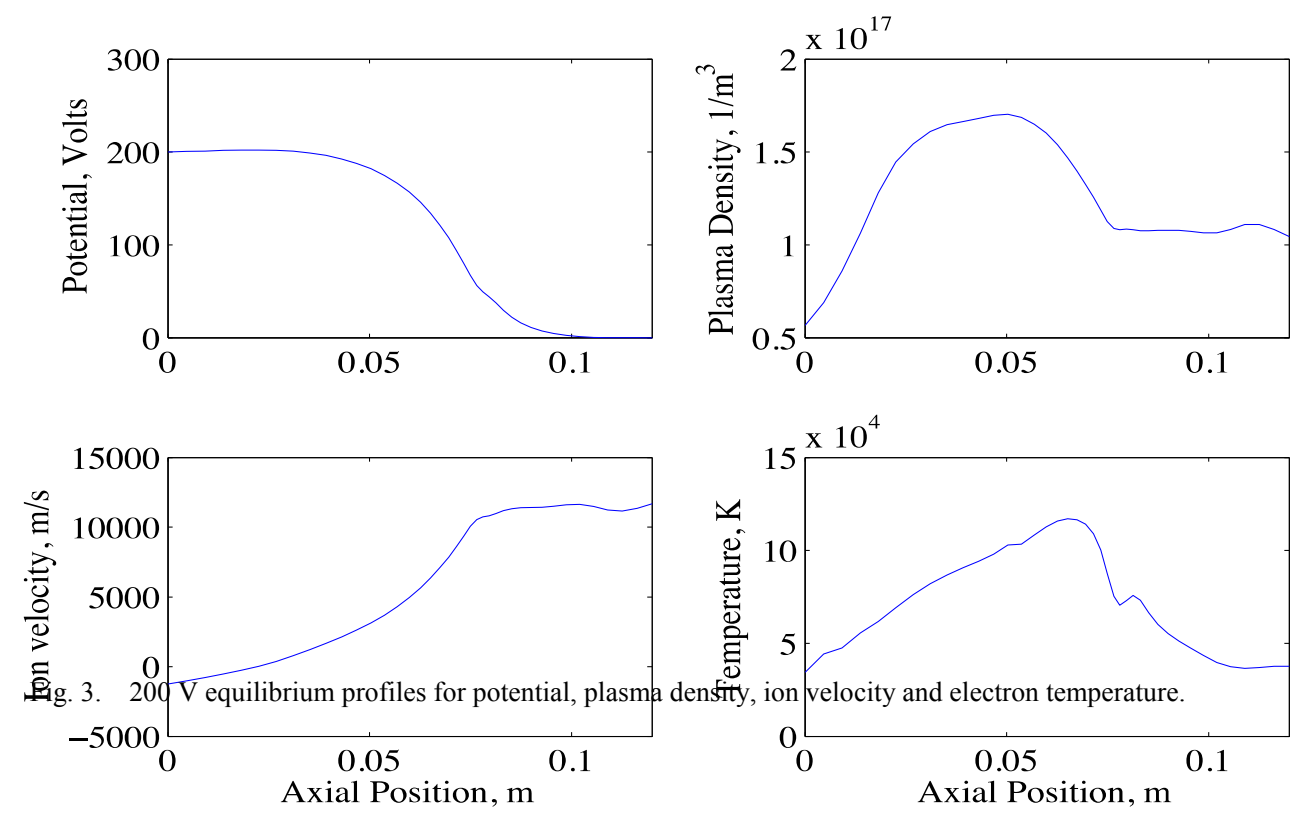

While the plasma is sustained, the time average discharge current is around .8 amps, substantially below the experimentally measured average value of $2.5 \mathrm{amps}$. Recall that there is no ad-hoc transport parameter used in the simulation, nor wall effects, and, therefore, the obtained current is derived solely from collisional transport and fluctuations generated within the plasma. Further discussion on the issue of electron transport is postponed until subsection 3.3 - for now we just note the relatively low discharge current predicted by the model.

Time-averaged 1D profiles (averaged over the azimuthal direction) for the plasma potential, ion velocity, plasma density, and electron temperature are given in Fig. 3. Such equilibrium profiles are in qualitative agreement with experiment. ${ }^{2}$ For example, the electric potential has the expected drop in the high magnetic field region (the peak in the magnetic field is at around $\mathrm{z}=.077 \mathrm{~m}$, just inside the channel exit at $\mathrm{z}=.08 \mathrm{~m}$, with the anode being at $\mathrm{z}=0$ ). The temperature peaks slightly upstream of the peak of the electric field, as a result of the strong joule-heating source. The ionization rate depends on the temperature, and not surprisingly it peaks slightly upstream of the temperature peak (not shown).

The nominal values of the maxima in the profiles are typically within a factor of two of the experimental values. We note that the electron temperature is about $10 \mathrm{eV}$ (compared to $15 \mathrm{eV}$ experimentally), which may be counterintuitive since one would expect the lack of wall losses to over predict the temperature. We believe the under predicted value results from too low of an electron current resulting from an overall low mobility. Consequently, the joule heating source term in the energy equation is not sufficient to drive up the temperature to the experimental values. The latter results in a low value for the plasma density, which the simulation under predicts by more than a factor of two.

The simulation profiles are also broader than those found experimentally. ${ }^{2)}$ We believe this is due to the mobility generated by the simulation, which has a broader shape than the experimentally measured mobility; the latter is known to have a strong, localized dip around the high magnetic field region, approaching classical values. ${ }^{2)}$ Such dip localizes the profiles, in particular the temperature profile as the effective thermal diffusivity is now severely reduced. In the simulation, the mobility dips in the magnetic field region but it is not nearly as localized, resulting in broader electric potential and electron temperature profiles, which in turn result in broader ionization rate and plasma density profiles. The effect of the electron mobility on the plasma equilibrium has also been clearly demonstrated in r-z hybrid models, where the choice of electron transport parameter (mobility model) largely determines the shape and location of the various profiles. ${ }^{7)}$

The profiles in Fig. 3 demonstrate that the model recovers the main plasma flow predicted theoretically and observed experimentally: a near anode region of ion back flow, followed by an ionization region, followed by an acceleration region. ${ }^{11)}$ The reversed ion flow results from a negative axial electric field in the anode region, spanning about $2 \mathrm{~cm}$. We note that in this region current continuity cannot be established with the negative electric field: electron pressure provides the needed force to push the electrons along towards the anode (and against the electric field), consistent the strong gradient in plasma density as the plasma moves from the anode region into the ionization region. On the other hand, the absence of wall effects does result in some unrealistic results. For instance, note how the plot of the plasma density is fairly flat downstream of the channel exit. In the r-z hybrid models the plasma density drops as we move downstream of the channel exit owing to the expansion of plasma in the radial direction. Since the model does not have radial losses the plasma density downstream is roughly constant, consistent with an ion beam moving with a fairly constant exiting speed. 


\subsection{Fluctuations}

\section{Linear regime}

We wish to differentiate fluctuations in the "linear regime" from those that are sustained in the saturated, turbulent state. By linear regime we refer to the regime where fluctuations, growing out of a smooth equilibrium, have small amplitude so that nonlinear coupling and saturation effects are negligible. Present theoretical analysis of Hall thruster instabilities is largely limited to linear analysis, so it is in this regime where comparison with theory is most appropriate. After all, theoretical work in plasma turbulence has shown that a number of nonlinear coupling effects, including nonlinear frequency shifts as well as nonlinear excitation of linearly damped normal modes, can result in turbulence that deviates markedly from linear stability predictions. With the above in mind, in this subsection we examine fluctuations that arise at the beginning of a typical simulation, before amplitudes become large and before a saturated state is achieved. A typical hybrid simulation begins with an initial background of neutral and ion superparticles that form an initial state. The loading of particles is done so that the particle densities are smooth, other than the small, intrinsic noise associated with the PIC treatment. Soon after starting the simulation - in about one microsecond we observe the 2D profile of plasma density shown in Fig. 4. We note that disturbances localized downstream of the channel exit (at $\mathrm{z}=.08$ ) emerge from the smooth background. Given the strong axial localization we claim the instability is gradient driven. In order to test this hypothesis we use the linear instability criterion for long wavelength drift modes derived by Frias et al., ${ }^{12)}$ which is similar to the original theory given by Esipchuk. ${ }^{13)}$ In Frias' theory, gradients in the magnetic field and the plasma density, characterized by scale lengths $L_{B}$ and $L_{n}$ respectively, can trigger instability. A necessary condition for instability is given by: ${ }^{12}$

$$
\left(e E / T_{e}+2 / L_{B}\right)\left(1 / L_{n}-2 / L_{B}\right)>1 /\left(4 \rho_{s}^{2}\right)
$$

where $\rho_{s}$ is the ion-sound Larmor radius. In our simulations, the right-hand side of the inequality above is small, so instability is effectively dictated by the sign of each of the two factors in the left-hand side of the inequality. In Fig. 4 we plot the product of these two factors as a function of axial position, with instability taking place when the product is positive. From the figure we note that is it negative for most of the channel except for a region downstream of the peak in the magnetic field (at around $\mathrm{z}=.08 \mathrm{~cm}$ ). In this region, the second factor is positive due to $-2 / L_{B}$ being positive ( $L_{B}$ being negative as the magnetic field decreases downstream of its peak). The first factor is also positive since the electric field is large and positive here and thus the first term within the factor, namely $e E / T_{e}$, dominates the negative second term $\left(2 / L_{B}\right)$. We note

a rather good agreement between the instability criterion prediction and the location of the emergence of the fluctuations in the hybrid simulation, giving support to the claim that the fluctuations are gradient driven. The instability thus occurs in the negative gradient magnetic field region near the channel exit. Frias' theory shows that the azimuthal propagation is in the positive EXB direction with a phase speed much lower than the EXB electron drift, both of which are corroborated in the simulations. Furthermore, his analysis shows that the imaginary part of the mode frequency (growth rate) is larger than the real part (propagation frequency). The latter is also born out of the simulation results, as movies of the disturbances depict them growing faster than they propagate.
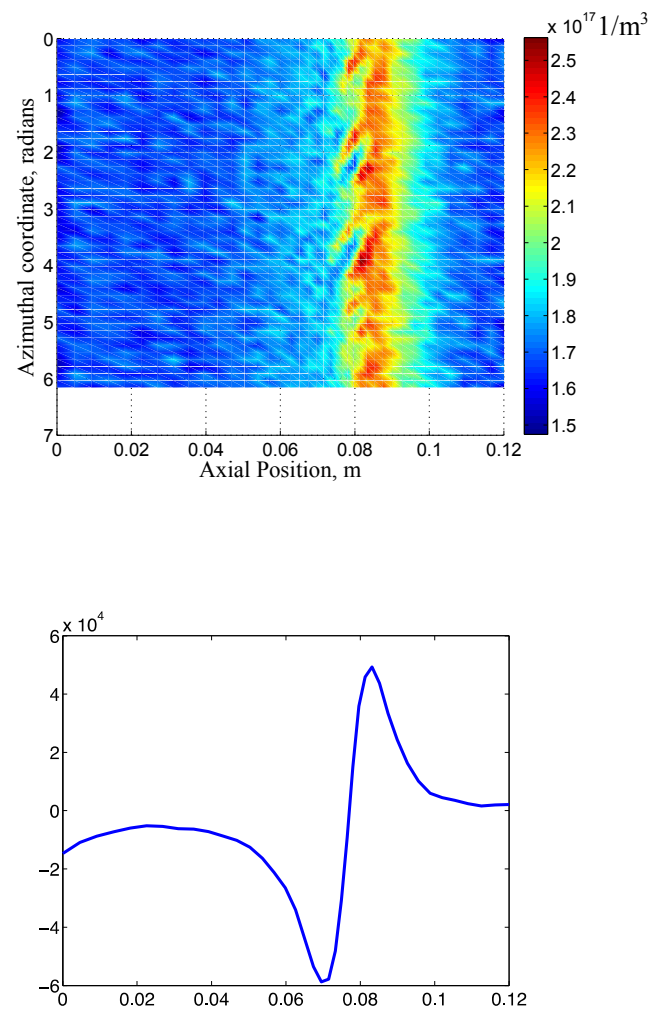

Fig. 4. Top plot: 2D map of the plasma density, with the vertical axis corresponding to the azimuthal direction and the horizontal axis to the axial direction. Bottom plot: stability factor as a function of axial position. Note the structures in the top plot emerge where the stability factor is positive, corresponding to linear instability. 
Two more tests were done to further verify the waves as gradient driven waves. First, a simulation started with the external magnetic field in the opposite direction results in disturbances drifting in the opposite azimuthal direction, consistent with the reversal of the EXB direction. Secondly, a simulation started with ionization turned off results in a wave pattern emerging in the same location, with no apparent difference from the case where the ionization was not removed. These two tests show that the fluctuations are indeed of the drift type and that ionization is not responsible for these waves, and together with the instability criterion prediction, lend support to Frias' theoretical prediction for gradient driven, drift instability in Hall thrusters. Interestingly, in the SHT these waves are unstable around the channel exit, unlike in other thrusters where the waves are shown to be unstable in either the anode region or in the plume region. ${ }^{14)}$

\section{Saturated regime}

A. Breathing mode.

In this section, we examine the disturbances that are generated by the simulation in the steady state regime. Since the objective is to resolve low frequency, long wavelength phenomena, the simulation must span enough time that the lowest frequency mode in the system, the so-called "breathing mode", is resolved. Indeed, the most ubiquitous oscillation in Hall thruster plasmas is the breathing mode. It is an ionization driven mode whereby the disparate timescales involving neutral gas advection and avalanche ionization result in relaxation oscillations in discharge current as well as an upstream/downstream shifting of the ionization front. The mode, long observed experimentally, was first recovered in hybrid fluid-PIC simulations by Fife, ${ }^{5)}$ giving much impetus to hybrid modeling efforts. Since then, the breathing mode has been recovered in other simulation models, both fluid and kinetic. The mode was first understood using a simple zerodimensional predator-prey model proposed by Fife, but its prediction of the frequency scaling as $\sqrt{\mathrm{v}_{\mathrm{n}} \mathrm{V}_{\mathrm{i}}} / \mathrm{L}$ seemed to differ from simulation results observed by Fernandez ${ }^{15)}$ and Boeuf, ${ }^{16)}$ which yielded a frequency scaling given by $\mathrm{v}_{n} / \mathrm{L}$ instead. As explained by Barral ${ }^{17)}$ with a theoretical model that successfully incorporates all of the key physics, the latter scaling corresponds to the more prevalent, high amplitude nonlinear mode frequency scaling, with the former scaling only applying to low amplitude, "linear modes". The breathing mode frequency obtained in our model (from the discharge current in Fig. 2) is around $3 \mathrm{kHz}$. This frequency is consistent with the nonlinear mode scaling frequency, $\mathrm{f} \sim \frac{\mathrm{v}_{\mathrm{n}}}{\mathrm{L}} \sim \frac{250 \mathrm{~m} / \mathrm{s}}{.07 \mathrm{~m}} \sim \frac{3600}{\mathrm{sec}}$. (Barral's theoretical analysis gives a more precise expression for the frequency, but the frequency for our simulation does not change appreciably from the simple $\mathrm{f} \sim \frac{\mathrm{v}_{\mathrm{n}}}{\mathrm{L}}$ estimate. $)^{17)}$

We note that the relatively low $3 \mathrm{kHz}$ frequency above derives from a somewhat long ionization region, resulting from rather broad equilibrium profiles (see Fig. 3). The experimental profiles, as mentioned above, tend to be more peaked, in particular, the electron temperature profile (which directly impacts the ionization rate profile and thus the breathing mode frequency). Finally, with the breathing mode on the order of a few $\mathrm{kHz}$, the simulation must run on the order of milliseconds. Previous work with this model did not quite resolve the breathing mode timescale, which in turn implies that the equilibrium supporting higher frequency waves was not a true steady state equilibrium. ${ }^{8)}$ Since mode instability criteria generally depend on the established equilibrium, resolving the latter is necessary in order to assess the instability and dispersion properties of fluctuations.

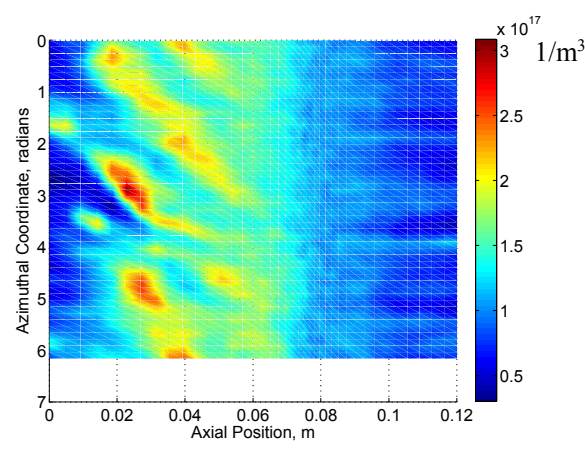

Fig. 5. 2D snapshot of the plasma density in the saturated regime.
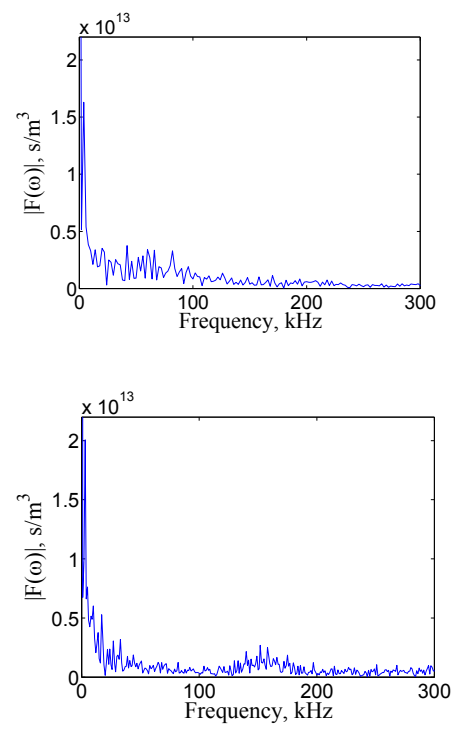

Fig. 6. Frequency spectrum for an upstream density mode (top plot), and a downstream density mode (bottom plot).

\section{B. Higher frequency waves}

Disturbances of the order of $50 \mathrm{kHz}$ are readily observed in the simulations. Fig. 5 shows a 2D snapshot of the plasma 
density for our nominal 200 -volt simulation. In the figure, we note broad, extended fluctuations spanning about a third of the channel. We believe these are ionization driven drift modes as they are predominant in the ionization zone. As noted in previous works, drift modes in Hall thrusters propagate in the EXB electron drift direction, but with a propagation speed of only a fraction of the EXB velocity. In our case, the phase velocity is a few thousand meters per second.

It is worth noting that these disturbances are not purely azimuthal but are tilted, with a component of their wavenumber vector towards the anode. The fluctuations, unlike the coherent breathing mode, are somewhat broadband. Their dominant azimuthal wave number is low $(m \approx 3,4)$ and thus similar to the so-called rotating spoke instabilities. ${ }^{18)}$ In Fig. 5 we also note a transition region of rather quiescent wave activity around the strong magnetic field region (at around 0.08 $\mathrm{m}$ ). Downstream of this region, axial wave structures emerge as observed in Fig. 5. Such waves are also broadband, but are of higher frequency as compared with the ionization driven waves upstream. The Fourier spectrum of the fluctuations for an upstream plasma density point and a downstream point are shown in Fig. 6. (The peak at very low frequency corresponds to the breathing mode.) We note that upstream there is wave power centered at around $60 \mathrm{kHz}$, while downstream we have wave power at a higher frequency (note the feature at around $150 \mathrm{kHz}$ ). The downstream, axial waves propagate outwards and have a phase velocity comparable with the ion velocity. Waves with such dispersion are referred to in the literature as "transit time" "19) waves, and from the linear regime analysis they are most likely gradient driven waves.

\subsection{Electron transport}

The axial electron current generated in the simulation is proportional to the product of the plasma density and the axial electron velocity. Fluctuation-induced transport results from the correlation $\tilde{n}_{e} \tilde{u}_{e z}$. In this paper, we study the contribution of azimuthal disturbances to the transport arising from the $4^{\text {th }}$ and $5^{\text {th }}$ terms in Eq. (1), that is, from fluctuating EXB and diamagnetic drifts. As mentioned in the introduction, such terms are absent in r-z hybrid models. In Fig. 7 we plot the plasma density and electric potential at a grid point upstream in the channel, where the broad, ionization fluctuations mentioned above are prevalent. We note in the figure that the plasma density and electric potential exhibit a marked correlation indeed these are transport-producing fluctuations. However, it is important to note that the phase between the electric potential and the plasma density is close to zero (a zero phase difference would imply no transport as the velocity and density would then be out of phase, resulting in no time average current contribution). As a result the overall transport produced by these fluctuations is small, certainly smaller than that which would derive from a more favorable phase (the rather unfavorable phase for transport contrasts with the more favorable density-potential phase found experimentally in spoke instabilities) ${ }^{18)}$. We also note (not shown) that the transport arising from the diamagnetic azimuthal disturbance ( $5^{\text {th }}$ term in Eq. (1)) is zero due to its phase difference with the plasma density. Therefore, the azimuthal disturbances result in fluctuation-induced transport only via the $4^{\text {th }}$ term in Eqn. 1. We can compare the transport that arises from such azimuthal disturbances from the remaining contributions (the first three
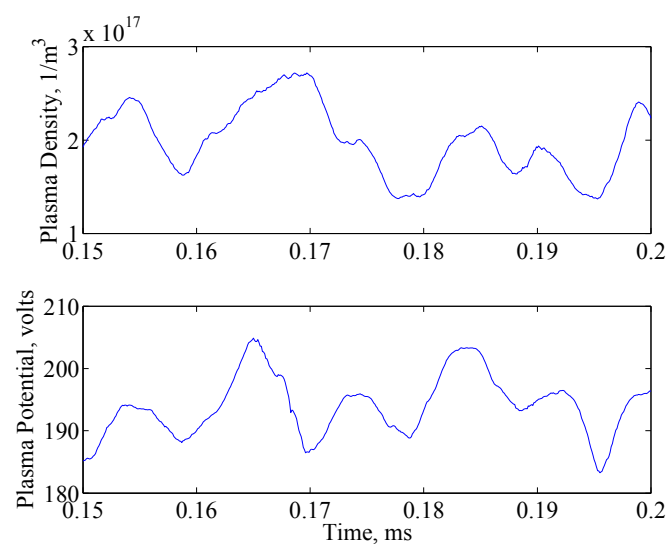

Fig. 7. Evolution of plasma density (top plot) and plasma potential (bottom plot).

terms in Eq. (1)), which we now refer to as "classical contribution". This is shown in Fig. 8, where the percentages of the total current coming from the azimuthal disturbances and classical contribution are shown. We note that fluctuationinduced transport dominates the rest of the transport in the ionization region of the discharge. It also dominates in the far downstream region. On the other hand, in the acceleration region where the magnetic field is strong the fluctuationinduced transport is subdominant. Note that at the anode $(\mathrm{z}=0)$ and at the downstream boundary $(\mathrm{z}=0.12)$ the contribution is effectively classical due to the boundary condition of constant electric potential in those two boundary planes.

In Fig. 8 we also plot the EXB shear of the electron flow as it has been speculated that the EXB shear may be partly responsible for the reduction in fluctuation-driven transport in the Hall thruster, much as it is responsible for turbulent transport reduction in fusion devices. ${ }^{20)}$ As it is evident from the plot, the shear profile is sharply (double) peaked in a region around $z=0.08$. However, the region over which the fluctuationinduced contribution is subdominant extends over a far larger span, from about $\mathrm{z}=5 \mathrm{~cm}$ to $\mathrm{z}=10 \mathrm{~cm}$. While shear may play a role (as it hinted by the local feature in the fluctuation 
contribution at around $\mathrm{z}=0.08$, see Fig. 8), it does not seem to explain the transport profiles.

\section{Conclusions}

This paper has presented our recent progress with an axial/azimuthal hybrid simulation for Hall thrusters. Our simple model has been applied to the SHT geometry and has been able to recover a plasma equilibrium that is qualitatively consistent with experimental measurements. Fluctuations
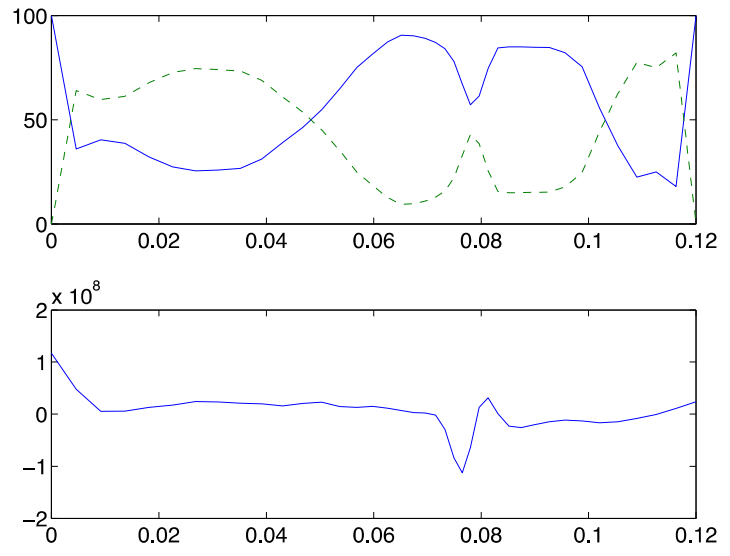

Fig. 8. Top plot: Contribution of classical and fluctuation-induced transport as a function of axial position (solid trace classical, dashed trace fluctuation-induced). Bottom plot: EXB shear rate as a function of axial position.

have been studied in the low-amplitude, linear regime as well as in the saturated regimes. In the linear regime drift modes emerge, driven by gradients in plasma density and magnetic field, consistent with Frias's linear stability analysis. At saturation, the simulation has yielded a number of fluctuations, including a) the ubiquitous low-frequency breathing mode, b) medium frequency, tilted, ionization waves upstream in the channel and c) higher frequency, axial, outward propagating disturbances downstream. The fluctuations result in a contribution to electron transport that surpasses the "classical" contribution upstream and downstream of the acceleration region of the channel. On the other hand, such fluctuationinduced transport is not sufficient to drive enough current to match experimental measurements. The natural conclusion drawn from this study is that other modes, perhaps higher frequency kinetic instabilities, ${ }^{10)}$ may contribute more significantly to electron transport. Our simulations also suggest that the shear in the EXB electron flow does not seem to control the overall turbulent transport profile, although it may play a role in the local reduction of fluctuation levels.

\section{Acknowledgments}

The authors would like to acknowledge Mark Cappelli, Cheryl Lam, and Eunsun Cha for many fruitful discussions.

\section{References}

1) Janes, G. S. and Lowder, R. S.: Anomalous Electron Diffusion and Ion Acceleration in a Low-Density Plasma, Phys. Fluids, 9 (1966), 1115.

2) Meezan, N. B., Hargus, W.A., Jr. and Cappelli, M. A.: Anomalous electron mobility in a coaxial Hall discharge plasma, Physical Review, 63 (2001), No. 2, 026410.

3) Chouieri, E.: Plasma oscillations in Hall thrusters, Phys. PlasmasII, 8 (2001), 141.

4) Gascon, N, Meezan, N. and Cappelli, M.: Low Frequency Plasma Wave Dispersion and Propagation in Hall Thrusters, IEPC-000, 27 $7^{\text {th }}$ International Electric Propulsion Conference, Pasadena, CA, Oct.15-Oct. 19, 2001.

5) Fife, J. M.: Hybrid-PIC modeling and electrostatic probe survey of Hall thrusters, Ph.D. Dissertation, Dept. of Aeronautics and Astronautics, Massachusetts Inst. of Technology, Cambridge, MA, 1999.

6) Hagelaar, G. J. M, Bareilles, J., Garrigues, L. and Boeuf, J. -P.: Twodimensional model of a stationary plasma thruster, J. Appl. Phys., 91 (2002), No. 9, 5592.

7) Scharfe, M. K., Gascon, N., Cappelli, M. A. and Fernandez, E.: Comparison of Two Dimensional Hall Thruster Hybrid Model to Experimental Measurements, Physics of Plasmas, 13 (2006), 083505 .

8) Lam, C., Fernandez, E. and Cappelli, M.A.: A 2D Hybrid Hall Thruster Simulation that Resolves the EXB Electron Drift Direction, IEEE Transactions on Plasma Science, 43 (2015), No. 1, pp 86-94.

9) Hargus, W. A.: Investigation of the plasma acceleration mechanism within a coaxial Hall thruster, Ph.D. Disseration, Mechanical Engineering Dept., Stanford Univ., Stanford, CA, 2001.

10) Adam, J. C., Heron, A. and Laval, G.: Study of Stationary Plasma Thrusters Using Two-Dimensional Fully Kinetic Simulations, Physics of Plasmas, 11 (2004), 295.

11) Ahedo, E., Martinez-Cerezo, P. and Martinez-Sanchez, M.: One Dimensional Model of the Plasma Flow in a Hall Thruster, Physics of Plasmas, 8 (2001), 305.

12) Frias, W., Smolyakov, A., Kaganovich, I. D. and Raitses, Y.: Wavelength Gradient Drift Instability in Hall Plasma Devices. I Fluid Theory, Physics of Plasmas, 19 (2012), 072112.

13) Esipchuk, Y. V. and Tilinin, G. N.: Drift Instability in a Hallcurrent Plasma Accelerator, Sov. Phys. Tech. Phys., 21 (1976), 417.

14) Frias, W., Smolyakov, A., Kaganovich, I. D. and Raitses, Y.: Wavelength Gradient Drift Instability in Hall Plasma Devices. II Applications, Physics of Plasmas, 20 (2013), 052108.

15) Fernandez, E., Cappelli, M. A. and Mahesh, K.: 2D simulations of Hall thrusters, Center for Turbulence Research Annual Research Briefs, Stanford Univ., Stanford, CA. 1998, pp.81-90.

16) Boeuf, J. P. and Garrigues, L.: Low Frequency Oscillations in a 
E. FERNANDEZ et al.: Characterization of Fluctuations and Electron Transport in a Two-Dimensional Hall Thruster Model

Stationary Plasma Thruster, Journal of Applied Physics, 84 (1998), 3541 .

17) Barral, S. and Peradzynski, Z.: Ionization Oscillations in Hall Accelerators, Physics of Plasmas, 17 (2010), 014505.

18) Ellison, C. L., Raitses, Y. and Fisch, N. J.: Cross-field electron transport induced by a rotating spoke in a cylindrical Hall thruster, Physics of Plasmas, 19 (2012), 013503.
19) Barral, S., Makowski, K., Peradzynski, Z. and Dudeck, M.: Transit-Time Instability in Hall Thrusters, Physics of Plasmas, 12 (2005), 073504.

20) Burrel, K. H.: Effects of EXB Velocity Shear and Magnetic Shear on Turbulence and Transport in Magnetic Confinement Devices, Physics of Plasmas, 4 (1997), 1499. 